\title{
An embedded processing of differential pulse voltammetry (DPV) data using ARM processor (LPC1768)
}

\begin{abstract}
This paper reports an embedded system design of a digital signal processing (DSP) unit for an In-situ analyzing of electrochemical data obtained by differential pulse voltammetry (DPV) from a three-electrode cell. The system implements smoothing filter, multiple peak detection and Gaussian distribution algorithms has been developed onto ARM cortex-m3 processor (NXP LPC1768) in order for the DPV to be able of detecting and analyzing heavy metals for various applications. The DPV can be used to measure sample of concentrations and environmental monitoring without the need of personal computer (PC) or expert user. Potential scanning range of this device is between $-1500 \mathrm{mV}$ and $+1500 \mathrm{mV}$ within frequency range $0.1 \mathrm{~Hz}-1 \mathrm{kHz}$ which can be applied for several electrochemical components. The developed algorithms has been tested for measuring heavy metal samples with different concentration.
\end{abstract}

Keyword: Differential pulse voltammetry (DPV); Digital signal processing (DSP); Electrochemical instrument; LPC1768 